\title{
El monumento como un todo. El plan director de restauración
}

\author{
Monument like a whole. The restoration master plan
}

P. Latorre $^{(*)}$

\section{RESUMEN}

El objetivo del artículo es exponer los problemas que plantea en el trabajo de restauración el estudio de la arquitectura histórica siguiendo el paradigma mecanicista y causal, que entiende su estructura como un sistema cerrado y estable, con un comportamiento lineal y predecible, que se imagina acabado, intemporal y reversible; en contraposición, se propone acometer su estudio conjuntamente con el de sus usuarios y el medio natural y socio cultural en el que se sitúan como un sistema abierto, complejo y dinámico, con un comportamiento no lineal y holístico de los elementos y partes que lo componen, en continua interacción, transformación y evolución de su configuración y construcción y, en definitiva inestable e irreversible. Algunas de estas ideas se elaboraron durante la redacción y ejecución del plan director de restauración de la Catedral de Santa María de Vitoria-Gasteiz del que se han extraído las imágenes que ilustran el artículo.

\section{SUMMARY}

The aim of this paper is to present the problems that poses in the restoration works the study of historical architecture following the mechanistic paradigm and causal that understands its structure as a stable and closed system, with a linear and predictable behaviour, that is imagined immutable, timeless and reversible; we propose, in contradistinction, to undertake its study together with its users and the natural and social environment in which it is immersed, as an open, complex and dynamic system, with a non linear and holistic behaviour of the elements and parts that make it up in continuous interaction, transformation and evolution of its configuration and construction and definitely, unstable and irreversible. Some of these ideas were elaborated during the redaction and execution of the restoration master plan of the Santa Maria Cathedral of VitoriaGasteiz, from which the images that illustrate this article have been taken.
Palabras clave: Estudios previos; preexistencia; interdisciplinariedad; sistema; complejidad restauración.
Keywords: Previous studies; pre-existence; interdisciplinary; system; complexity; restoration. 
1. Foto aérea del casco histórico y de la ciudad de Vitoria-Gasteiz.

2. Los arcos codales contienen las enormes deformaciones de los pilares de la nave de la Catedral de Santa María de Vitoria antes de su eliminación en la restauración del arquitecto $\mathrm{M}$. Lorente en la década de los 60. Archivo del Territorio Histórico de Álava. DAF $(\mathrm{H})$ c.4, n61. PD, Imagen 57

3. Mujer tendiendo la ropa en uno de los patios interiores de las viviendas que se adosan en el lateral oriental de la Catedral de Santa María. Foto Quintas, Vitoria.
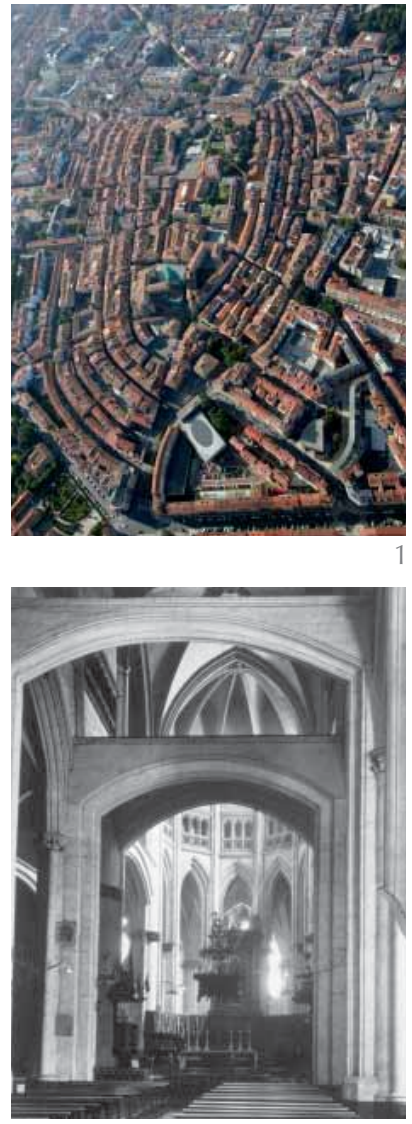

Arturo Rosenblueth Stearns, (1900, 1970), investigador, médico y fisiólogo mejicano.

${ }^{2}$ Norbert Wiener, $(1894,1964)$, matemático estadounidense, conocido como el fundador de la cibernética. Acuñó este término en su libro: Cibernética o el control y comunicación en animales y máquinas, publicado en 1948, del que se ha extraído la cita y que Wiener dedica a Rosenblueth "compañero durante muchos años de la ciencia".
"...El Dr. Rosenblueth" ha insistido siempre en que la exploración adecuada de estos espacios en blanco de la ciencia solo se puede realizar por un equipo de científicos, cada uno especialista en su propio campo pero poseyendo un conocimiento muy completo y disciplinado de sus vecinos y habituados todos al trabajo en equipo, $\mathrm{CO}^{-}$ nociendo las costumbres intelectuales de cada uno y reconociendo la importancia de una sugerencia nueva de un colega antes de haber decidido cuál sea la expresión definitiva. El matemático no necesita tener la pericia para dirigir un experimento fisiológico, pero debe tenerla para entenderlo, criticarlo o sugerirlo. El fisiólogo no necesita ser capaz de probar un determinado teorema matemático, pero debe serlo para captar su significado fisiológico y decir al matemático cómo debería enfocarlo. Hemos soñado durante años con una institución de científicos independientes, que trabajen en equipo en una de esas regiones apartadas de la ciencia, no como subordinados de un alto ejecutivo, sino unidos por el deseo, que es ciertamente necesidad espiritual, de comprender la región elegida como un todo y demostrarse recíprocamente la fuerza para tal comprensión...", Norbert Wiener ${ }^{2}$ (1).

\section{EVOLUCIÓN Y COMPLEJIDAD DE LA ARQUITECTURA HISTÓRICA Y SU CA- RÁCTER SISTÉMICO}

"Si consideramos que la evolución implica un proceso de cambio acumulativo, las ciudades pueden identificarse con metabolismos que van adaptándose en el tiempo para sobrevivir..." (2) (Figura 1).

Es evidente que las ciudades y los edificios -como estructuras inorgánicas- no evolucionan, ni van modificándose por sí mismos como las especies de la naturaleza siguiendo procesos de selección natural. Sin embargo, al igual que las ciudades de las que forman parte, la arquitectura como consecuencia de su interacción con el medio en el que se encuentra inmersa se ve sometida por el grupo social que la utiliza a un proceso continuo y acumulativo en el tiempo de cambios y transformación de su construcción y configuración para adecuarla en cada momento a sus necesidades.

Las razones por las que la arquitectura se deteriora, pierde su funcionalidad o su valoración social o cultural son múltiples. Por un lado, los materiales y la construcción van erosionándose y deteriorándose por la acción de los agentes atmosféricos y la actividad biológica, que acaban provocando su pérdida de cohesión y resistencia. A este deterioro se superponen los esfuerzos que deben soportar los materiales y los elementos constructivos para mantener en equilibrio la estructura, cuyo efecto se ve multiplicado por la degradación normal de éstos. Además, existe una agresión mucho más traumática provocada por catástrofes naturales o que tiene su origen en la acción humana. Tanto la degradación de los materiales y la construcción, como el colapso parcial de algún elemento o cuerpo de la edificación, dificultan o impiden el desarrollo normal de su función o pueden poner en peligro su estabilidad, lo que provocará la reparación de las partes deterioradas y la reconstrucción de las colapsadas (Figura 2).

Por otro lado, la evolución y transformación normal del medio natural o urbano $y$, sobre todo, del socio- cultural y económico en el que se ve inmerso el grupo social que ocupa el edificio modificará también sus usos, costumbres y preferencias. Con el paso del tiempo irá también variando su estructura en número, actividad, origen, etc. lo que irá mermando la funcionalidad de la arquitectura que deberá someterse a una obra de rehabilitación que modifique su configuración, la distribución de sus espacios o la reforma de sus instalaciones para responder a las nuevas demandas de uso (Figura 3).

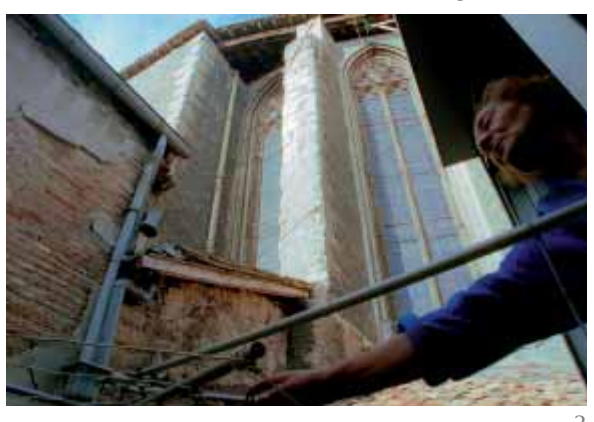

Este proceso de transformación del grupo de usuarios, igualmente disminuirá la capacidad de la arquitectura para satisfacer determinadas expectativas de carácter estético o representativo y la pérdida de su valoración social, cultural o simbólica conducirá a una reforma o remodelación que actualice las formas periclitadas y dote al edificio de otras nuevas acorde con los gustos existentes y la necesidad de representación.

Por lo general, los cambios descritos son lentos, lo que provoca que vayan acumulándose pequeñas deficiencias que van superándose con los trabajos imprescindibles de mantenimiento. Estas pequeñas obras de reparación provocan modificaciones casi imperceptibles que, al ir sucediéndose de forma continua y acumulativa a lo largo del tiempo, pueden llegar a suponer transformaciones significativas (Figura 4).

A pesar de estas pequeñas intervenciones, la acción constante del medio natural y so- 


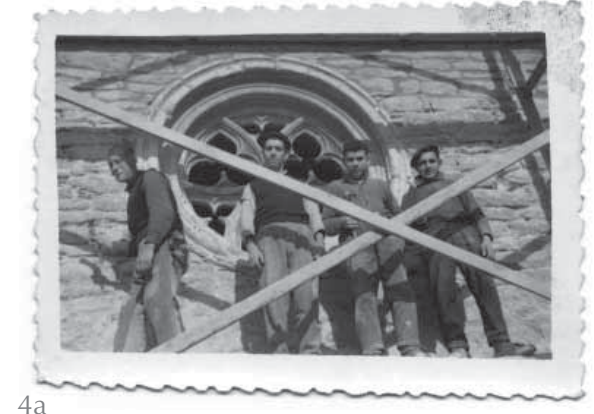

$4 \mathrm{a}$

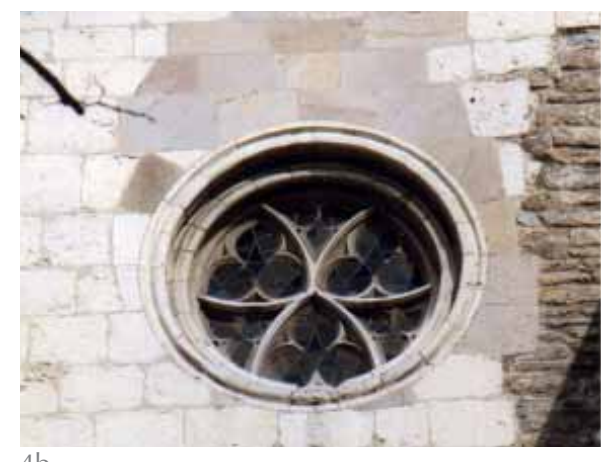

$4 \mathrm{~b}$

ciocultural va inexorablemente degradando la arquitectura, al mismo tiempo que va transformando al grupo de usuarios, lo que provoca el deterioro de la construcción, la pérdida de funcionalidad o de valor simbólico que van superponiéndose en el tiempo hasta un punto en el que es inevitable una reforma importante de su configuración. Las grandes transformaciones de una arquitectura coinciden generalmente con hitos importantes en la historia de los usuarios y en el contexto social, cultural y económico y se enmarcan entre múltiples obras de mantenimiento que dan respuesta al funcionamiento habitual del mismo. Cada una de las grandes intervenciones que sufre una arquitectura le permite recuperar su valor y estimación social abriendo un nuevo periodo de uso que garantiza su pervivencia en el tiempo y la continuidad de su evolución (Figura 5).

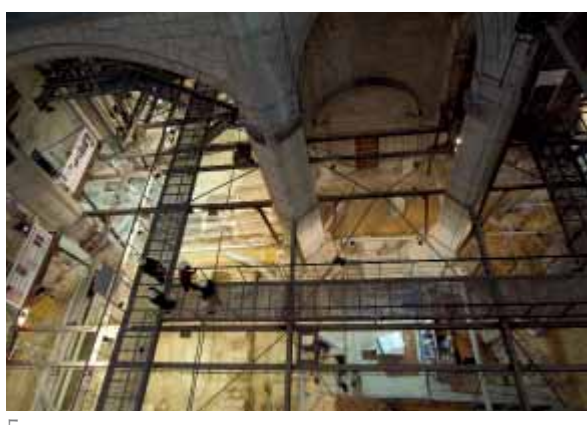

En definitiva, la arquitectura se construye para satisfacer determinadas necesidades del grupo social que la promueve y deberá cumplir unos objetivos de carácter formal, constructivo o funcional determinados por el promotor y adaptarse a las preexistencias del medio sobre el que se construirá. Una vez construida su propia materialidad condicionará los comportamientos de los usua- rios y el desarrollo de su actividad, tanto si satisface sus expectativas cómo si incumple los objetivos que impulsaron su construcción. Simultáneamente, la arquitectura y sus usuarios se verán condicionados por el medio en el que se encuentran inmersos, al que tendrán que someterse adaptándose a sus propias condiciones cambiantes que provocarán la degradación de la construcción y la transformación del grupo de usuarios. Finalmente, cuando la arquitectura no responda a las expectativas de carácter funcional, constructivo o formal serán los usuarios los que promoverán su modificación para adecuarla a sus nuevas necesidades y demandas.

Este proceso de interacción continuo entre la arquitectura y sus usuarios, entre éstos y el medio natural y sociocultural, y entre éste y la arquitectura configura un bucle de relaciones que se realimenta siguiendo un proceso iterativo en el tiempo que provoca la complejidad de su configuración y patología. Por este motivo, la estructura arquitectónica y el grupo social que la ocupa, interaccionando entre ellos y con el medio constituyen un sistema complejo, abierto y dinámico de múltiples elementos y partes interrelacionados, interaccionando y en constante transformación, evolución y cambio $(3)^{3}$.

Comprender la arquitectura histórica formando parte de un sistema impide que podamos reducir su estudio exclusivamente al de los elementos que componen su estructura, obviando el conocimiento del grupo social que la ocupa, del medio en el que se encuentran inmersos y de las relaciones e interacciones que se producen entre ellos. Para comprender este sistema es imprescindible no solo estudiar conjuntamente todos las partes y elementos que lo componen (estructura), sino también la totalidad de relaciones e interacciones que se producen entre ellos (comportamiento), entendiendo

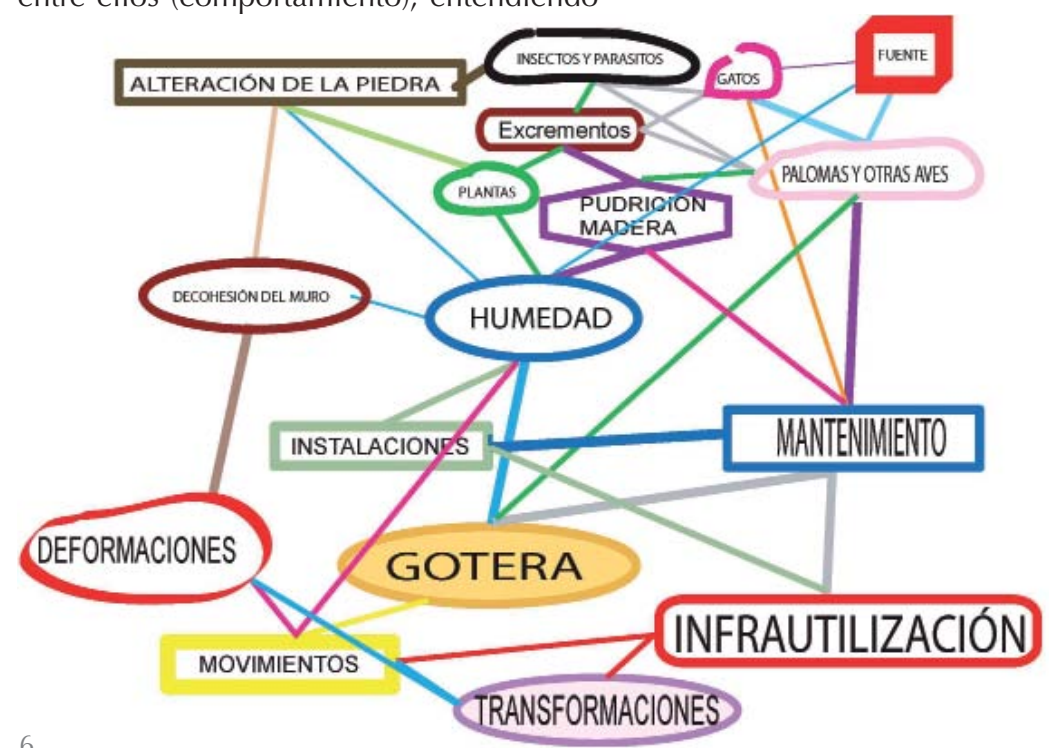

4a. Grupo de trabajadores durante la restauración de $M$. Lorente, junto a uno de los ventanales el muro meridional de la Catedral de Vitoria. (Foto: Pérez Ugarte) 4b. Nuevo ventanal circular copiando la tipología del anterior realizado durante la misma restauración.

5. El interior de la Catedral de Vitoria durante el actual proceso de restauración, 2008. Sistema de acodalamientos, apeos y pasarelas para permitir los trabajos de excavación arqueológica, recalces de la cimentación y la visita guiada.

6. Esquema de las interacciones que se establecen entre diversos elementos que forman parte del sistema en el que se integra una arquitectura histórica para analizar el origen de una gotera. góticos circulares restaurados en
3 «... Un sistema entendido como un todo no es un objeto sino una manera de ver un objeto. Reside en un fenómeno holístico que sólo puede ser entendido como producto de la interacción entre las partes...» 
que forman un todo con un comportamiento holístico y complejo, del que emergen de forma, muchas veces imprevisible, otros nuevos que no pueden explicarse a través del estudio aislado de cada una de las partes y elementos resultantes de su descomposición (4) ${ }^{4}$ (Figura 6).

\section{DEL MODELO MECANICISTA Y CAU- SAL AL MODELO CAÓTICO: LA ESTRA- TEGIA DE LA INVESTIGACIÓN}

"...nos hemos vuelto deterministas absolutos y aun los que quieren conservar los derechos del libre arbitrio humano, dejan por lo menos al determinismo reinar sin división en el mundo inorgánico... Una pequeñísima causa, que escapa a nuestra percepción, determina un efecto considerable que no podemos ignorar, y entonces decimos que el efecto se debe al azar. Si conociésemos bien las leyes de la naturaleza, y la situación del universo en el momento inicial, conseguiríamos predecir exactamente su situación en un momento posterior. Pero, incluso en el caso de que las leyes naturales no nos ocultasen sus secretos, continuaríamos sabiendo su situación sólo de manera aproximada. Si esto nos facultase a pronosticar la situación sucesiva con la misma aproximación, no necesitaríamos más, y afirmaríamos que el fenómeno se vaticinó y que las leyes gobiernan todo. Sin embargo, no ocurre siempre así; puede suceder que pequeñas diferencias en las condiciones iniciales provoquen diferencias enormes en el fenómeno definitivo. Un leve error en las primeras se convertirá en uno colosal en el segundo. Se hace imposible predecir..." (5).

Uno de los problemas más importantes de la ciencia y, en general, de todos nuestros hechos cotidianos es saber interpretar la realidad que nos rodea. No todos somos capaces de entender y deducir las mismas cosas al ver lo mismo, ya que la capacidad de ver y comprender determinados fenómenos depende directamente de la formación y experiencia del observador. El arquitecto -como todo especialista- al abordar la investigación de una arquitectura histórica sigue la metodología de proyectos para investigar y comprender los mecanismos del proceso creativo que han permitido a su predecesor definir su diseño, "transmutándose" con él para entender sus modos de trabajo. Siguiendo esta metodología, la mayoría de los estudios previos que se elaboran de los monumentos para su restauración restituyen la arquitectura que se está estudiando como si se estuviese elaborando el proyecto que permitió su ejecución, extrayendo directamente de la realidad los datos que permitirían reproducirla, construyéndola ex novo.
Por ejemplo, al observar en la realidad un arco de medio punto o una bóveda de cañón sabemos que su directriz es semicircular, porque ésta es la forma con la que se diseñan estos elementos constructivos $y$, de acuerdo a esta percepción -prefijada y aprendida previamente- se definirá su geometría, lo que conducirá a registrar en la realidad únicamente los datos necesarios (centro y radio) para restituir la directriz semicircular. Del mismo modo, se idealizará el aparejo de su construcción para analizar el comportamiento constructivo y estructural de un arco tipo, cuyos resultados se extenderán a todos los arcos de la misma forma y tipología, que serán igualmente idealizados de acuerdo a su diseño constructivo (6).

El modelo obtenido siguiendo este proceso puede someterse -igual que el proyecto de una arquitectura inexistente- a las leyes de la mecánica para calcular y estimar su comportamiento, que lógicamente debería coincidir o aproximarse al que presenta el edificio en la realidad. Utilizaremos este modelo para ensayar con él, como si estuviésemos elaborando su proyecto, tratando de comprobar los defectos que presenta su diseño y que provocan ahora la patología que hemos detectado. La comparación entre el comportamiento del modelo y la realidad nos permitirá elaborar un diagnóstico que determine y explique estos "errores" y diseñar el proyecto de restauración que los corrija.

Sin embargo, en la realidad la geometría que poseerá el arco de una arquitectura histórica-aunque no sea perceptible- se asemejará más al perfil irregular de una naranja y presentará probablemente algún tipo de fisura entre sus juntas o la fractura de alguna de las piezas que lo configuran. Su materialidad, en el instante mismo en el que realizamos su análisis, responderá no sólo a su comportamiento como estructura en el tiempo, sino también a como lo han hecho sus apoyos en muros o pilares y éstos en su cimentación, que descansará sobre un suelo concreto. De hecho, sólo a través del análisis minucioso y detallado del sistema formado por el arco, sus apoyos, la cimentación y el suelo, y la de éstos con respecto a los elementos constructivos adyacentes integrados en el conjunto de la edificación, podremos comprender su verdadero comportamiento y patología y buscar las causas que han provocado la deformación existente. En este proceso, hay que evitar generalizar y trasladar los datos extraídos de un elemento constructivo concreto a todos los semejantes. Muchas veces, aunque los materiales, forma y tipología de varios elementos parezcan idénticos, su comportamiento y evolución podrá diferir en el tiempo, ya que las condiciones de contorno serán es- 
pecíficas para cada uno de ellos en función de la posición que ocupen en el espacio del edificio (Figura 7).

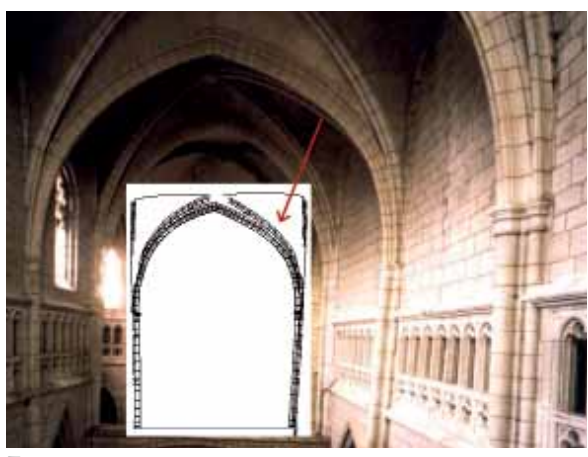

En la práctica, el comportamiento definido a través del cálculo utilizando modelos idealizados de una arquitectura histórica, carecerá de la fiabilidad necesaria y casi con seguridad los resultados no podrán asimilarse con el que ha tenido el edificio, que responderá a una realidad mucho más compleja. Por ejemplo, las deformaciones y tensiones que se obtienen en el cálculo por elementos finitos de modelos simétricos y regularizados de la realidad darán resultados simétricos, cuando en la realidad difícilmente lo son, ya que nunca lo será la propia estructura, ni por supuesto sus condiciones de contorno. Por otro lado, es inevitable que al elaborar el diagnóstico de la patología que presenta un edificio inconscientemente se distorsione la interpretación de los hechos de la realidad para adecuarla al comportamiento que predicen los cálculos del modelo que se ha elaborado y que muchos datos y hechos que se están produciendo sean ignorados o ni siquiera percibidos, dadas las limitaciones de la propia formación y capacidad del observador $(7)^{5}$. Sabemos que la historia de la restauración está plagada de actuaciones fracasadas o especialmente desafortunadas con la arquitectura que se pretendía rescatar, al abordar su estudio siguiendo las pautas de comportamiento de un modelo idealizado elaborado con las herramientas y la metodología habitual de la historia y el proyecto de arquitectura (Figura 8).

En definitiva, la búsqueda de la atemporalidad de la arquitectura construida y la sublimación de la idea y del proceso creativo del arquitecto en un contexto histórico concreto, tropiezan en el trabajo de restauración con una realidad temporal donde el cambio, la superposición y la evolución son continuos. Por este motivo, el estudio de la arquitectura para su restauración debe acometerse conjuntamente con el de sus usuarios y el medio natural y socio cultural en el que se sitúan, analizándolos y entendiendo que configuran en la realidad un sistema abierto, complejo y dinámico, do- tado de un elevado número de elementos y partes interrelacionados en continua interacción, que presenta un comportamiento holístico e inestable que deriva en un proceso de cambio y evolución continuo, que es irreversible.

Como consecuencia de la inestabilidad y de la no linealidad de su comportamiento el sistema es muy sensible a las modificaciones de su equilibrio y cualquier cambio en alguna de sus partes tendrá con seguridad repercusiones en otras. De este modo, cualquier intervención que modifique la configuración o las relaciones entre los elementos y partes del sistema en el que se integra una arquitectura histórica puede provocar de forma imprevisible una nueva patología. Por este motivo, el éxito del diagnóstico y de las propuestas de restauración están claramente condicionadas por la capacidad para prever el comportamiento y la reacción que tendrá el monumento una vez materializadas las soluciones del proyecto y la fiabilidad de esta predicción supeditada a la dependencia sensitiva que el comportamiento de un sistema tiene a las condiciones iniciales de su configuración.

\section{EL CARÁCTER PLURIDISCIPLINAR DE LOS ESTUDIOS, EL EQUIPO MUL- TIDISCIPLINARIO, LA INTERDISCI- PLINARIEDAD DEL MÉTODO Y DEL DIAGNÓSTICO $Y$ LA TRANSVERSALI- DAD DE LA RESTAURACIÓN}

"...En los últimos años se ha hablado mucho del dialogo entre disciplinas como fuente de inspiración de nuevos problemas y nuevas soluciones. Para el estudio de la complejidad éste es uno de los elementos fundamentales, ya que, aunque cuente con metodologías varias, su objeto de estudio abarca problemas relacionados tanto con las ciencias llamadas duras como con las blandas. Sistemas complejos existen en biología, en química, en física, en sociología, en economía, etc. Se habla mucho, sin entender muchas veces de lo que se está hablando, de interdisciplinariedad, de multidiciplinariedad, de plusridisciplinariedad, de transdisciplinariedad, con frecuencia usándolos como sinónimos, cuando cada uno de estos términos tiene significados precisos muy diferentes. Sin embargo, sigue faltando el verdadero diálogo entre disciplinas necesario para el avance en el conocimiento de sistemas complejos en particular y de la ciencia en general..." (8).

El conocimiento de la realidad material del monumento, de los usuarios y su entorno, entendidos como partes de un sistema, exige un cambio de metodología de investigación y su estudio debe ejecutarse acudien-
7. Vista del transepto de la Catedral de Vitoria desde el lateral norte. Fotogrametría del alzado de uno de los arcos perpiaños.

8. Alzado interior del muro occidental del crucero norte de la Catedral de Vitoria y detalle del triforio que lo recorre. El elemento constructivo no coincide con el elemento artístico que está seccionado por una interface de corte entre dos fases de la construcción. A la izquierda la decoración de la barandilla cuadrilobulada del triforio está calada $(\mathrm{A})$ y a la derecha ciega (B)

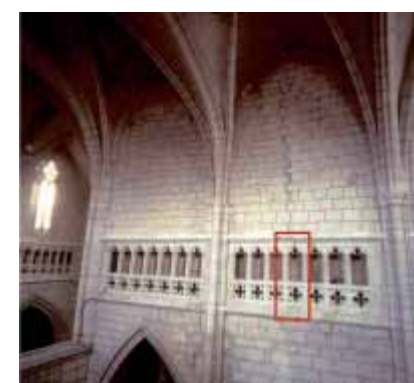

$8 a$

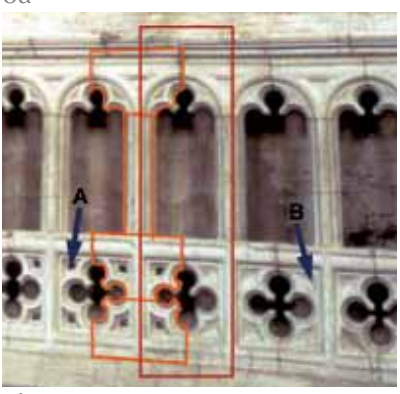

$8 b$

${ }^{5}$ «... ¿Ahora comprende? Usted no ha observado, a pesar de haber visto. Eso es lo que quería decirle. Ahora bien, yo sé que hay diecisiete escalones, porque he visto $y$ he observado..

...Este es un asunto realmente misterioso - comenté-. ¿Qué cree que puede significar?

- No tengo datos todavía. Es un error capital tratar de formular teorías antes de tener datos. Insensiblemente, uno empieza a retorcer los hechos para que se adapten a las teorías, en luga de que las teorías se adapten a los hechos...» 
9. Esquema: Proceso para la restauración. do a todas las disciplinas con capacidad para aportar datos e información sobre el mismo. Esta investigación debe adquirir un carácter pluridisciplinar y abordarse desde campos tan diversos como la geología, biología, química, física, ingeniería, arquitectura, historia, arqueología, geografía, antropología, etc.

Además, la restauración de una arquitectura histórica no depende de una actuación estrictamente arquitectónica y es imprescindible acometer simultáneamente intervenciones de carácter social, biológico, urbanístico, medioambiental, arqueológico, jurídico, etc. Normalmente, este tipo de intervenciones se escapan de nuestro ámbito de actuación y, por supuesto, de nuestros conocimientos, comprensión y capacidad para darles una solución dentro de los cauces habituales en los que se mueve la restauración arquitectónica. Por este motivo, es necesario formar equipos multidisciplinarios de especialistas con una experiencia probada, la tecnología adecuada, un conocimiento suficiente de las disciplinas y de las técnicas utilizados por sus colegas en su campo específico y habituados a trabajar en equipo; de tal modo, que los resultados obtenidos por cada especialista puedan ser comprendidos y utilizados por sus compañeros en su propia investigación (Figura 9).

Pero la creación de equipos multidisciplinarios no es una condición suficiente para garantizar el éxito de la investigación, ya que es probable que sin una dirección adecuada cada especialista actúe individualmente estudiando los problemas que afectan exclusivamente a su disciplina, lo que generará una acumulación de datos y puntos de vista diferentes sin ninguna relación entre ellos que acabarán resultando inútiles por insuficientes, irrelevantes o imprecisos. Además, es frecuente que muchas de estas disciplinas no tengan desarrollada una tecnología específica para la investiga-

FASE I: Acciones ANTES de la Restauración

1-A. DESCUBRIMIENTO, RECONOCIMIENTO Y PROTECCIÓN

1-B. PROYECTO DE ESTUDIOS Y OBRAS DE CONSERVACIÓN Y EMERGENCIA

\begin{tabular}{l|l} 
- PLURIDISCIPLINAR & 2. INVESTIGACIÓN Y CONOCIMIENTO DEL OBJETO, DE SU CONTEXTO Y ENTORNO \\
- INTERDISCIPLINAR & $\begin{array}{r}\text { 3. PATOLOGÍA Y DIAGNÓSTICO. EL PLAN DIRECTOR DE RESTAURACIÓN } \\
\text { - TRANSVERSAL }\end{array}$ \\
\hline & 4. A PLAN DE VIABILIDAD Y REDACCIÓN DE PROYECTOS DE RESTAURACIÓN \\
\hline & 5-A. PLAN DE COMUNICACIÓN Y DIFUSIÓN \\
\hline & FASE II: Acciones DURANTE la Restauración
\end{tabular}

4-B. EJECUCIÓN DE OBRAS DE RESTAURACIÓN

5-B. COMUNICACIÓN Y EXPOSICIÓN, DIDÁCTICA, DIFUSIÓN Y DIVULGACIÓN FASE III: Acciones POSTERIORES a la Restauración

6. PUESTA EN USO Y DESARROLLO DEL PLAN DE VIABILIDAD.

7. PLAN DE MANTENIMIENTO Y CONSERVACIÓN ción de la arquitectura, ni especialistas experimentados en estas aplicaciones con la capacidad para interpretar correctamente los datos obtenidos, lo que acabará provocando que los resultados de estos estudios apenas tengan incidencia en las decisiones de proyecto, generando muchas dudas su financiación.

Para alcanzar el éxito en la investigación de la arquitectura histórica es imprescindible conseguir la perfecta coordinación de todos los especialistas y la adopción de una estricta metodología de trabajo interdisciplinar que habilite un procedimiento para el intercambio real de información entre especialidades que permita relaborar los ensayos y objetivos de cada estudio en función de los resultados que van obteniéndose en cada fase de la investigación. Metodológicamente, es necesario establecer un procedimiento de ida y vuelta permanente entre el modelo y la realidad que nos obligue constantemente a comparar los datos obtenidos en los diferentes estudios para establecer las relaciones que se producen entre ellos, de tal modo, que ante cualquier contradicción podamos acudir siempre a la realidad para obtener nuevos datos con los que poder ofrecer finalmente una verdadera síntesis integradora que permita la confirmación o falsación de las hipótesis planteadas en el diagnóstico.

Lamentablemente, la progresiva especialización a la que nos condenan las disciplinas de las diferentes ramas del conocimiento con el objetivo de obtener la máxima eficacia en su aplicación y desarrollo, obliga a la investigación a situarse normalmente en compartimentos estancos de los que es muy difícil salir para observar y conocer el trabajo de otros investigadores $y$, sobre todo, para desarrollar trabajos en equipos multidisciplinarios que sigan y apliquen una metodología verdaderamente interdisciplinar. N. Wiener comenta que son precisamente las áreas abandonadas como tierra de nadie, entre varios campos de diferentes disciplinas, las más eficaces para la investigación, el desarrollo científico y el trabajo en equipo, lamentándose que la ciencia está en manos cada vez más especializadas que únicamente exploran campos reducidos y compartimentados.

Aunque en el proceso de análisis y estudio de la arquitectura histórica se habla continuamente del trabajo en equipo y de la necesidad de integrar los conocimientos necesarios para su investigación, lo cierto es que la realidad del trabajo habitual con el que nos enfrentamos los profesionales de la restauración está muy lejos del carácter pluridisciplinar que se supone deberían te- 
ner los estudios previos $y$, sobre todo, de la interdisciplinariedad metodológica necesaria para establecer un diagnóstico correcto, que carece a menudo del rigor necesario al desarrollarse a partir de una realidad idealizada que sigue una metodología claramente mono disciplinar que conduce inevitablemente a una restauración "sin claros objetivos, inútil e incluso dañina para el bien cultural" (9). De hecho, este monográfico reclama precisamente la "integración" de la multiplicidad de conocimientos que confluyen en el trabajo de restauración. No es muy difícil deducir que cuando se reclama algo, simplemente es porque no se está produciendo o se entiende que se está realizando de forma defectuosa.

En el trabajo de restauración es imprescindible plantearse la importancia que metodológicamente tiene realizar los estudios previos necesarios para permitirnos realizar un diagnóstico correcto en el que se recojan todos los problemas existentes, incluso aquellos que prevemos puedan presentarse en el futuro. Será toda la información recabada en los estudios y el diagnóstico realizado los que permitirán establecer adecuadamente los objetivos del proyecto de restauración, en el que habrá que diseñar las soluciones que permitan eliminar las interacciones negativas detectadas $\mathrm{o}$, si esto es imposible, minimizar o neutralizar sus efectos en la "construcción" del edificio. Un diagnóstico inadecuado o parcial nos llevará a proyectar soluciones incorrectas que pueden ser irreversibles. Además, si no se prevén la totalidad de los problemas que plantea el edificio histórico en un instante concreto y acometemos una obra de restauración de un modo lineal, para solucionar sólo determinados aspectos que nos hemos planteado, podremos hipotecar en el futuro la ejecución de la solución que hubiese sido la más idónea. Por eso, decimos que el proyecto de restauración debe ser transver$\mathrm{sal}^{6}$, acometiendo y solucionando de forma integral con una única solución de proyecto la totalidad de los problemas que plantea el edificio en un momento concreto.

Es importante entender que la mayoría de las veces será imposible corregir los errores de nuestras decisiones, ya que la realidad cambia en cada instante como consecuencia de nuestras acciones o simplemente como consecuencia de la existencia misma. Una decisión equivocada habrá transformado la realidad y nos impedirá corregir el error en el mismo sentido y contexto. Nada será igual que en el instante precedente, ya que la supuesta irreversibilidad de nuestras actuaciones no es más que una quimera que se verá arrastrada por la evolución constante del medio y los usuarios. Com- probado el fracaso de la solución ejecutada, sólo será posible realizar un nuevo diagnóstico de la realidad presente y proyectar una nueva actuación -corrigiendo la precedente- para alcanzar el objetivo que nos hemos propuesto, siempre y cuando el mismo error cometido no impida definitivamente la materialización de la solución correcta.

\section{EL MODELO PARA LA DIAGNOSIS Y SU ELABORACIÓN. EL PLAN DIREC- TOR DE RESTAURACIÓN}

\begin{abstract}
"...La ciencia clásica ha sido fundamentalmente reduccionista, en el sentido de asumir como axioma metodológico básico el que para estudiar un objeto lo que había que hacer era reducirlo a sus partes y estudiarlas aisladamente. Una vez conocidas las propiedades de las partes, las propiedades del sistema se desprenden por sí solas. Se trataba, por tanto, de reducir el estudio de un sistema a su análisis, a su disección. Este principio analítico ha sido enormemente fecundo, en particular en física. Sin embargo, desde las disciplinas que se ocupan del estudio de objetos de una cierta complejidad pronto se empezó a presentir su insuficiencia. Tan importante como la disección y el análisis es la integración y la síntesis..." (10).
\end{abstract}

Es en este contexto en el que hay que entender la importancia que tiene la investigación y estudio del estado actual del monumento (11), ya que sólo a partir de la elaboración de un modelo o representación de éste podremos prever el resultado de la restauración y, por tanto, imaginarla y diseñarla. Además, sólo a través del estudio y análisis de este modelo podremos deducir una hipótesis de cómo fue su diseño "original" que utilizaremos, comparándolo con el estado que presenta en la actualidad, para definir la evolución de su configuración, construcción, patología, etc. a lo largo del tiempo. Es muy importante advertir que este trabajo de investigación no debe convertirse en un registro notarial y documental del monumento para cumplir el trámite preceptivo exigido por las diferentes cartas y normativas de protección del patrimonio. El objetivo de los estudios previos no es realizar una copia detallada y exacta del monumento, sino elaborar un modelo del sistema en el que se integra la arquitectura que describa los aspectos de su realidad material y de las relaciones que definen la dinámica de su comportamiento en el tiempo, que permitan explicar el origen y las causas de la patología que presenta. Es imprescindible en este proceso de investigación dotarse de un modelo de comportamiento correcto que nos permita prever la reacción que tendrá el monumento una vez materializadas las soluciones diseñadas en la propuesta de restauración, de tal modo,
Aunque muy común en la literatura científica el término transdisciplinar no aparece en el diccionario de la RAE y hemos preferido utilizar la palabra transversal y el concepto de transversalidad de la intervención. 

modelo para la diagnosis.

11. Perspectiva del modelo fotogramétrico 3D completo del interior de la Catedral de Vitoria. PD, p.100.
10. Esquema: Elaboración de

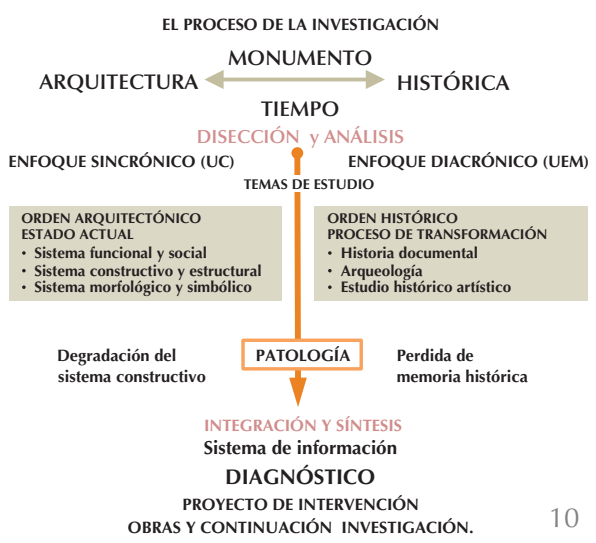

que podamos en este proceso ir corrigiendo estas propuestas hasta conseguir una respuesta idónea que garantice una nueva etapa en su evolución (Figura 10).

Con este objetivo, los estudios previos deben partir de una disección de las partes que componen el sistema (arquitectura, usuarios y medio) en los elementos que los configuran para realizar su análisis y comprensión y a partir de su definición establecer las relaciones e interacciones que se producen entre ellos. A la hora de abordar la descomposición de la arquitectura es necesario entender que su estructura -como

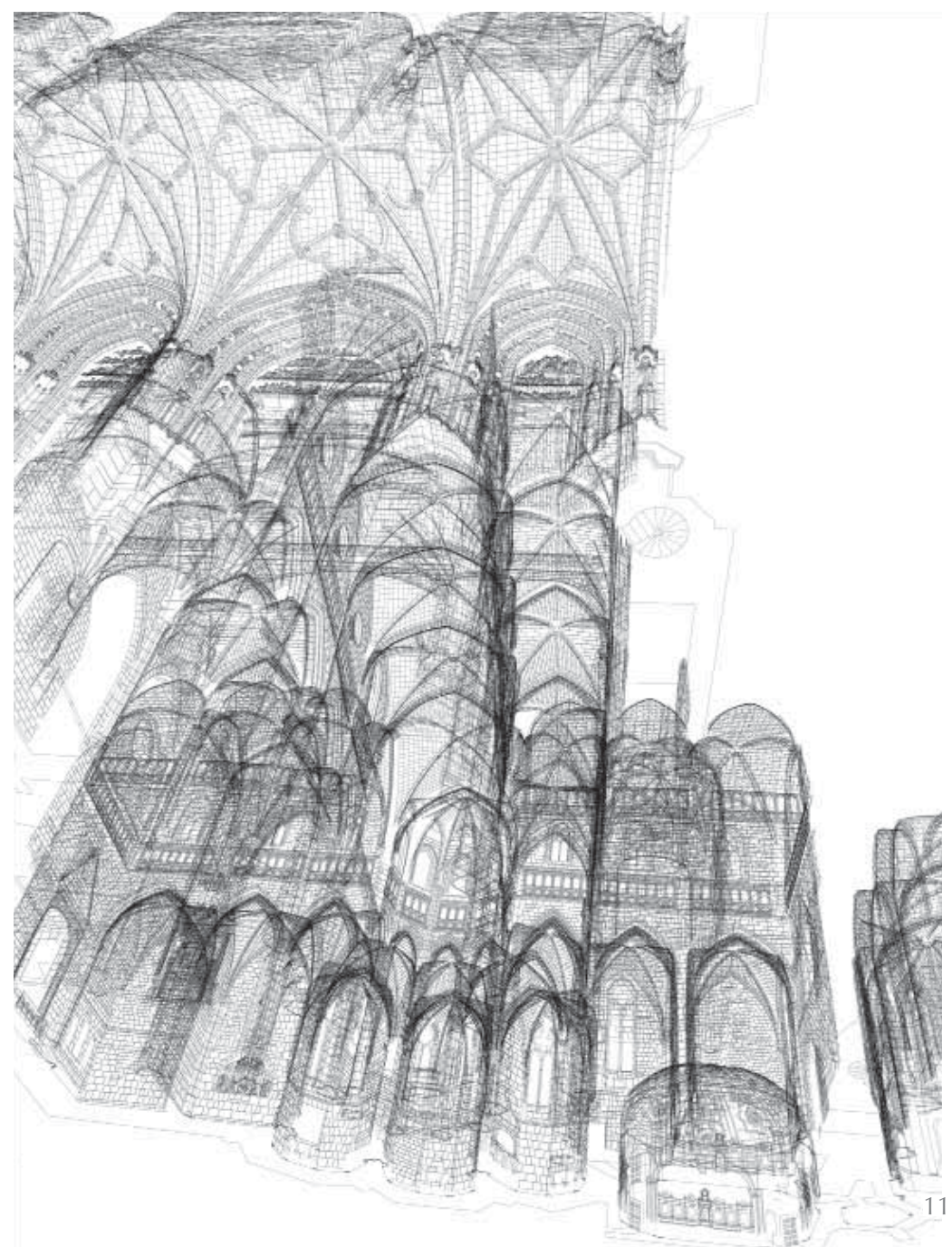

consecuencia de su propia evolución- es el resultado de la concatenación de múltiples arquitecturas que van sucediéndose y superponiéndose constructivamente sobre el mismo espacio a lo largo del tiempo (12). Cada reforma configura una nueva arquitectura en la que la estructura previa (preexistencias) una vez modificada, se integra en la estructura resultante, que se configura y diseña a partir de los nuevos objetivos (formales, funcionales y constructivos) y las nuevas condiciones impuestas (socioculturales y económicas) y que la dotan de una nueva unidad que le confiere su condición sincrónica y arquitectónica. Simultáneamente, su estructura está configurada con las partes de las arquitecturas precedentes de cada una de las transformaciones que ha sufrido y que se han ido conservando a lo largo del tiempo, reutilizadas en la estructura de arquitecturas posteriores y que le permiten documentar el proceso histórico de su evolución y le confieren su condición diacrónica e histórica (13) (Figura 11).

Desde la condición sincrónica analizaremos la configuración real que presenta la estructura arquitectónica en el instante que iniciamos los estudios, entendiéndola en sus aspectos constructivos, formales y funcionales, relacionándola con su entorno físico y social y definiendo la patología que les afecta. Con este objetivo debemos descomponerla para su análisis en los elementos constructivos UC (Unidad o elemento Constructivo) que configuran los espacios que componen los diferentes cuerpos de su edificación. Cada uno de estos elementos dotará de características específicas para su análisis a cada uno de los materiales que los componen, tanto desde el punto de vista de su forma, colocación en la construcción o de la función del espacio que delimitan.

De este modo, la investigación de la arquitectura deberá iniciarse desde del estudio y conocimiento de las características de los materiales constructivos (sillar, mampuesto, viga, correa, etc.) que componen cada elemento constructivo y la del suelo en el que se asienta, definiendo en el laboratorio -a partir de muestras extraídas in situ- su caracterización y composición mineralógica y sus propiedades físicas, mecánicas y químicas, etc. A continuación tendremos que definir la forma y dimensiones específicas de cada material y el modo en el que se aparejan componiendo cada uno de las UC (muro, pilar, estribo, contrafuerte, arco, bóveda, cercha, faldón de cubierta, etc.) y cómo, de la unión de éstos, se llegan a conformar las diferentes partes de la construcción (cabecera, crucero, nave, capilla, torreón, etc.) hasta alcanzar la configuración completa de su estructura, tal y como ha llegado hasta nosotros (Figura 12). 
Una vez definidos estos elementos y los materiales que los componen habrá que estudiar el edificio en su conjunto, como organismo arquitectónico, con sus características formales, funcionales y constructivas específicas. Tendremos que relacionar el modelo obtenido con el medio en el que se encuentra inmerso, estableciendo desde las lesiones y degradaciones que presentan los materiales de modo individual, hasta los problemas y patologías más generales del comportamiento mecánico de su estructura o de la calidad de sus espacios, su capacidad para responder a las demandas funcionales que se le exigen, etc. Será el momento de realizar todo tipo de evaluaciones numéricas como las evaluaciones de carga, el estudio de las deformaciones, etc. y de concretar, en cartografías temáticas, la distribución y localización específica de las patologías detectadas, cruzando datos y comportamientos, asociando zonas y patologías hasta establecer conclusiones de los fenómenos y procesos observados.

Pero no podemos analizar sólo la arquitectura por unas características que suponemos tuvo en un instante del pasado y entenderla como si estuviese congelada en el instante mismo de su construcción, es necesario conocer el proceso histórico que ha provocado su transformación en el tiempo, ya que es este proceso el que nos permitirá entender sus problemas, las imperfecciones de su diseño al verse expuesta al medio específico en la que se sitúa y conocer como ha sido su respuesta para adaptarse al mismo y llegar hasta nosotros con la configuración que presenta en la actualidad. Existe una relación directa entre la configuración "estratificada" de su construcción y la heterogeneidad de su arquitectura en un instante y el proceso histórico que la ha modelado, entre su historia y su construcción y entre éstas y la historia del grupo social que ha promovido las transformaciones, etc. Desde la condición diacrónica analizaremos la estructura del monumento como el resultado de este proceso de transformación en el tiempo relacionado con su historia constructiva y social, de la que tendremos que deducir la configuración de cada una de las fases que ha tenido su estructura.

Con este objetivo, el estudio histórico y arqueológico del edificio se iniciará también partiendo del material, de su forma y características, de los procesos de su fabricación y manufactura, del modo en que se apareja, etc. Para abordar este análisis tendremos que descomponer la estructura conservada agrupando y delimitando todos los materiales coetáneos -es decir colocados en la misma fase histórica- que se encuentran rodeados de materiales de fases diferentes mediante relaciones de antero-posterioridad y delimitados por interfaces de corte o superposición constructiva y que se ha designado como UEM (Unidad Estratigráfica de Muro) $(14)^{7}$.

Todas las UEM coetáneas de una arquitectura histórica y todas las que pertenecen a las fases precedentes configuran la estructura conservada de la arquitectura de una fase específica del proceso de transformación que tratamos de definir. Por tanto, eliminando las UEM de las fases posteriores a ésta podremos plantear una hipótesis sobre cómo fue su arquitectura, simplemente rellenando en la propuesta las lagunas existentes a partir de las evidencias que aporta la estructura conservada, los restos arqueológicos de las zonas desaparecidas, los datos que pueda aportar el vaciado y estudio crítico de las fuentes documentales e iconográficas y las similitudes tipológicas que desde la historia de la arquitectura se puedan establecer de otros edificios coetáneos (15). Finalmente, planteadas las hipótesis de su configuración en cada una de las fases identificadas, podremos plantear cómo ha sido su proceso de transformación en el tiempo, desde el momento mismo de su ideación hasta el que adquirió el que presenta en la actualidad (Figura 13).

Paralelamente a este trabajo, será necesario estudiar la evolución de su patología como estructura, comparándola en cada momento con las hipótesis de las fases constructivas que hemos realizado, tratando de definir un desarrollo coherente entre ambas. Es posible, que la patología que podamos observar en la actualidad tuviese su origen en configuraciones diferentes del edificio y las anomalías detectadas procedan de otras configuraciones que dieron posteriormente lugar a transformaciones para corregirlas y estén "congeladas" en la actualidad (Figura 14)

Pero además, sobre estos problemas de carácter histórico, constructivo y estructural se superpondrán otros de índole artística, formal, simbólica o funcional igualmente complejos que tampoco debemos simplificar a través de modelos preconcebidos, integrando su comprensión en el proceso de transformación estudiado. Es imprescindible realizar una catalogación y una valoración histórico-artística del monumento, de los elementos artísticos y decorativos integrados en su arquitectura y del patrimonio mueble existente, contextualizándolos en el proceso histórico definido, estableciendo igualmente su patología (16). En paralelo, será necesario conocer los aspectos simbólicos de su arquitectura y de su patrimonio mueble y estudiar cual ha sido su evolución
7 El no 435 de Informes de la Construcción de 1995 fue la primera publicación en España dedicada con carácter monográfico a la disciplina designada como Arqueología de la Arquitectura. 

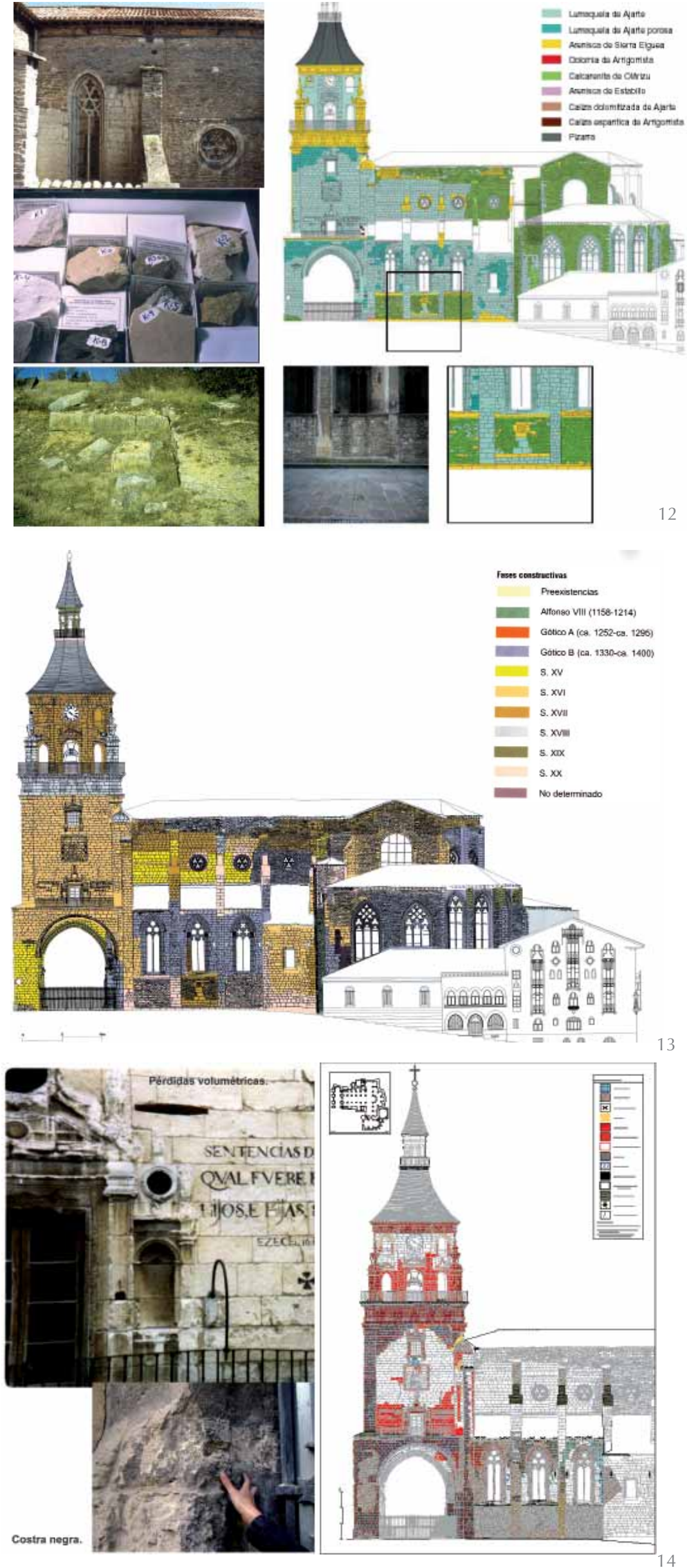

y, sobre todo, conocer su valoración actual en el contexto social existente.

También será necesario estudiar la evolución de los usos del edificio y conocer su relación con los espacios y las transformaciones definidas y establecer las necesidades del grupo social que lo utiliza, de modo que podamos abordar su actualización, dotándolo de las mejoras técnicas y de las instalaciones necesarias. Además, habrá que potenciar los aspectos divulgativos y de comunicación de sus valores patrimoniales y dotarlo de los elementos y espacios necesarios para permitir su visita cultural, compatibilizándola con su uso habitual.

Por último, es necesario entender el monumento en su entorno físico, social y cultural. Habrá que definir los elementos que configuran su entorno próximo y definen el paisaje que le rodea, sea éste rural o urbano. No podemos entender la actuación aislada de la estructura arquitectónica de su entorno más próximo que habrá que estudiar simultáneamente al edificio y con el mismo nivel de intensidad. También será necesario definir el proceso de transformación del entorno próximo con respecto al edificio integrándolos y estableciendo las interacciones y relaciones que existen entre ambos definiendo su patología, especialmente si se producen interacciones negativas que afectan a la conservación del monumento. Finalmente, habrá que definir y establecer medidas de protección de los elementos que configuran el paisaje a escala comarcal y proponer actuaciones para garantizar su conservación.

Con el modelo completamente definido y estructurado su desarrollo en el tiempo, estaremos en condiciones de proponer una hipótesis sobre el entorno socio-cultural y los planteamientos y condiciones que permitieron la primera construcción de nuestro edificio y los que sucesivamente provocaron, en los diferentes momentos de su historia, cada una de sus transformaciones. Podremos establecer todo tipo de estudios y comparaciones evolutivas de carácter tipológico de cada una de las fases que hemos definido, hasta llegar a su configuración actual y realizar un estudio de su evolución y desarrollo constructivo, formal, estilístico, artístico, espacial, tipológico, usos, proyectual, etc. Será el momento en que debemos estudiar el edificio, enmarcándolo y situándolo en su contexto histórico, en todos los niveles posibles, desde el marco más general de la historia de la arquitectura, el arte, la construcción, etc. hasta el más concreto de su propia historia y evolución. 
Evidentemente, la ejecución de todos estos estudios, su planificación y coordinación en el tiempo y la formación del equipo multidisciplinario que pueda abordar estos trabajos dotándolo de los mecanismos e infraestructura que permita su actuación coordinada y la aplicación de una metodología verdaderamente interdisciplinar, exige establecer una figura que vaya más allá del mero encargo de unos estudios y que contemple como objetivo la elaboración del modelo que hemos definido, la descripción de la patología existente, su diagnóstico y la elaboración de una serie de recomendaciones y propuestas de actuación que permita posteriormente gestionar de forma planificada la ejecución de todas las actuaciones diseñadas, tanto arquitectónicas como de otra índole que conduzcan a la transformación proyectada.

La figura que actualmente permite la contratación de un trabajo con este contenido es la del Plan Director de Restauración $(17)^{8}$. El objetivo de un Plan Director es elaborar el modelo del monumento al que nos hemos estado refiriendo, la formación del equipo multidisciplinario y la coordinación de su trabajo, la definición de los estudios necesarios y la gestión tanto del desarrollo del Plan Director como de la restauración. El trabajo debe iniciarse con la designación de un equipo de coordinación que deberá estar formado por lo menos por tres responsables de las tres áreas fundamentales de la investigación: historia y arqueología (-), conservación $(=)$ y arquitectura y restauración (+), que serán las responsables de decidir las demoliciones y excavaciones que vayan a efectuarse $(-)$, los tratamientos de conservación del material que permanezca (=) y cómo será la restauración y la arquitectura que se superponga (+) a la estructura conservada. En definitiva cada responsable decidirá el qué y el cómo de la materia que se elimina $(-)$, la que se conserva $(=)$ y la que se añade (+) (Figura 15).

De forma interdisciplinar se decidirán los temas de estudio que se abordarán en función de los problemas planteados y se consensuará la formación del equipo multidisciplinario. El número de componentes de este equipo nunca debe ser un listado cerrado y deberá ampliarse o reducirse en función de los resultados que vayan obteniéndose en el proceso de la investigación. Con el equipo ya formado, el trabajo deberá iniciarse con una recopilación de la documentación bibliográfica y del levantamiento existente. Con este material y una serie de inspecciones in situ se deberá redactar un primer proyecto en el que se incluyan todos los estudios y ensayos ne-
12. Mapa Litológico de la fachada principal de la Catedral de Vitoria. PD, p. 241

13. Mapa de la lectura estratigráfica de las fases constructivas de la fachada principal de la Catedral de Vitoria. PD, p. 180.

14. Mapa de patología y estados de alteración del material pétreo de la fachada principal de la Catedral de Vitoria. PD p. 383.

15. Proyecto de restauración de la Catedral de Vitoria. Sección del pórtico y la torre hacia el este. Latorre y Cámara, arquitectos.

${ }^{8}$ Algunas de estas ideas se elaboraron durante la redacción y ejecución del plan director de restauración de la Catedral de Santa María de VitoriaGasteiz del que se han extraído las imágenes que ilustran el artículo. Este trabajo fue galardonado con el Trofeo especial (primer premio) EUROPA NOSTRA AWARDS 2002 en la categoría de "Outstanding studies" "For the extensive and meticulous research of the Cathedral structure, as the basis for its restoration and for the study's stunning presentation"

www.europanostra.org/laureates-2002 www.catedralvitoria.com

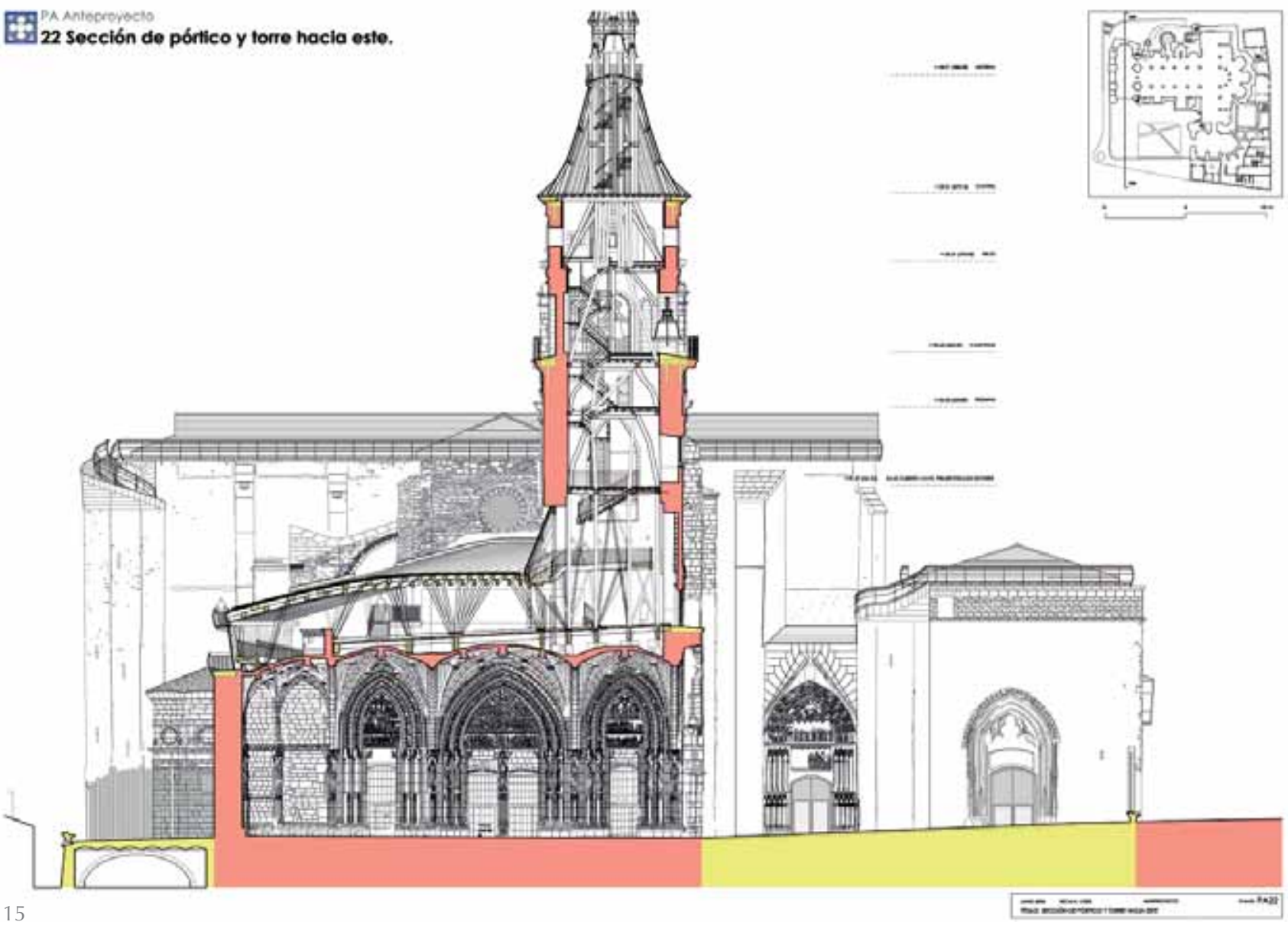


cesarios para la investigación, los medios auxiliares necesarios para llevarlos a cabo y las obras de limpieza, desescombro y de conservación imprescindibles para acometer el trabajo de investigación previsto en las condiciones de seguridad y accesibilidad idóneas. En el proyecto deberá incluirse un programa desarrollado en el tiempo de los estudios a realizar, ya que los resultados de determinadas investigaciones condicionarán la extensión y objetivos de otras. En el desarrollo de la ejecución de los ensayos previstos es fundamental crear equipos de trabajo en función de los temas de estudio planteados, de tal modo, que se garantice la coordinación y la interdisciplinariedad imprescindible para poder cruzar los datos y las explicaciones de cada espe- cialista a los resultados obtenidos, tratando de buscar la solución a los diferentes problemas detectados. Es en este punto en el que podrá abordarse como conclusión del trabajo, el diagnostico y las propuestas de actuación, incluyendo un programa planificado en el tiempo para su ejecución y un avance del presupuesto global, igualmente desglosado en anualidades según las posibilidades presupuestarias previstas por el promotor. Una vez concluida la redacción del documento, y tan importante como su elaboración, será la gestión y seguimiento que pueda hacerse de las propuestas que deberá abordar el desarrollo de los proyectos de restauración y el resto de actuaciones que se hayan previsto hasta la conclusión de la transformación proyectada.

\section{BIBLIOGRAFÍA}

(1) Wiener, N.: Cibernética, p.29, 1948, Madrid, 1960.

(2) Rogers, R.: La evolución de Londres, en Evolución, edición a cargo de Fabián, A.C., p. 105, 1988, Barcelona, 2001

(3) Alexander, C.: "Sistemas que generan sistemas". 3 Aspectos de matemática y diseño, p.63, 1964, Barcelona, 1969.

(4) Solé, R.: Redes complejas. Del genoma a internet. p.19, Barcelona, 2009.

(5) Poincare, H.: Ciencia y Método, p.58, 1908, Buenos Aires, 1946.

(6) Latorre, P.; Cámara, L.: "El levantamiento para la restauración: No hay método sin herramientas". Loggia, Arquitectu-ra \& Restauración, № 22-23, (2009), pp. 16-37.

(7) Conan Doyle, A.: Escándalo en Bohemia. Relatos breves recopilados en Las aventuras de Sherlock Holmes, 1891.

(8) León, M. y Sanjuán, M. A. F.: Las matemáticas y la física del caos, p. 209, Madrid, 2009.

(9) Muñoz, A.: "El estudio del monumento en el proyecto de intervención". Estudios Preliminares en la Restauración del Patrimonio Arquitectónico (2005), Madrid, pp. 7-24.

(10) Aracil, J. y Gordillo F.: Dinámica de sistemas, p.20, 1997, Madrid, 2007.

(11) Esteban, J.: "Estudios previos a la restauración de los monumentos". Restauración Arquitectónica (1992), Valladolid, pp. 159-177.

(12) Latorre, P., Cámara, L.: "Los procesos de transformación de la arquitectura en el tiempo. Consecuencias teóricas y metódológicas en el proyecto y la obra de restauración”. Quaderns Científics y Técnics de Restauració Monumental, nº 13 (2002), Barcelona, pp.161-175.

(13) Latorre, P. "La conservación del tiempo en la restauración: el proyecto estratigráfico" en las Actas del curso de Ar-queología aplicada al estudio e interpretación de edificios históricos, Instituto del Patrimonio Cultural de España, Ministerio de Cultura (2010), Madrid pp. 25-51.

(14) VV.AA.: "Leer el documento construido". Informes de la construcción, Vol.46 n 435 (1995).

(15) Latorre, P. y Caballero, L.: "Metodología e investigación del faro romano en la restauración de la Torre de Hércules de A Coruña (1990-1992)", Brigantium 20, (2009), pp. 193-227.

(16) Gómez Robles, L.: "Los valores del monumento restaurado. Una aproximación a la restauración científica". Revista ph 75 (2010), pp. 80-94.

(17) Azkarate, A.; Cámara, L.; Lasagabaster, J.I.; Latorre, P.: El Plan Director para la restauración de la Catedral de Santa María de Vitoria-Gasteiz, Vitoria, 2002. 